troposphere, starting from $3.6 \mathrm{~km}$, maintains a resolution of $150 \mathrm{~m}$. The "Atmospheric Data Processor" (ADP) is found to give an agreeable Doppler profile up to a height of $11 \mathrm{~km}$ where noise is less. Since the lower stratosphere extends from 10 to $20 \mathrm{~km}$ above the earth's surface, any spectrum estimating algorithm is expected to give good results in this particular range. There arises a need to go for a better functioning spectrum estimation method, especially at the height range of 14-17 km, as the existing methods [3-6] at this height fail to estimate the power spectrum accurately. Hence, semiparametric techniques can be focused on to improve the spectral estimation accuracy even for ill-conditioned data.

In this letter, vectors are represented using the lowercase bold letters and the matrices using the uppercase boldface. The scalars are indicated using the normal letters. Notations $|\cdot|,\|\cdot\|,(\cdot)^{*},(\cdot)^{T}$, and $E(\cdot)$ represents the modulus, Frobenius norm, Hermitian transpose (complex conjugate transpose), the transpose and the expectation respectively. The subscript [. $]_{k}$ denotes the vector $k$ th element, and $\boldsymbol{I}_{N}$ represents an identity matrix of order $\mathrm{N}$.

\section{Review of spice algorithm}

SPICE is a newly introduced technique for the sparse signal recovery based on the covariance fitting criterion. It is a hyper parameter free and gives better performance than other methods.

Let $y_{n}$ be the complex signal data, which is the weighted combination of $\mathrm{C}$ complex exponentials with frequencies

$\left\{\Omega_{r}\right\}_{r=1}^{N} \epsilon\left[0, \Omega_{\max }\right]$

$y_{n}=\sum_{r=1}^{C} q_{r} e^{j \omega_{r} t_{n}}+\varepsilon_{n}$

where $C$ is a constant, $\left\{t_{n}\right\}_{n=1}^{N}$ representing the instants of sampling time that can be irregularly spaced. The magnitude of the rth frequency component $\Omega_{r}$ is $q_{r}, \varepsilon\left(t_{n}\right)$ is the "additive white gaussian noise" component related to the $\mathrm{n}^{\text {th }}$ sampling time. Let $R \gg C$, be the number of frequency points that the frequency axis is sampled.

Then the complex signal can be replicated as

$\boldsymbol{y}=\sum_{r=1}^{R} \boldsymbol{d}\left(\omega_{r}\right) \mathrm{q}_{r}+\varepsilon$

where $\boldsymbol{y}=\left[y_{1}, y_{2}, \ldots, y_{N}\right]^{T}$

$$
\begin{gathered}
\boldsymbol{D}=\left[\boldsymbol{d}\left(\omega_{1}\right), \boldsymbol{d}\left(\omega_{2}\right), \ldots, \boldsymbol{d}\left(\omega_{N}\right)\right]^{T} \text { with } \\
\boldsymbol{d}\left(\omega_{r}\right)=\left[e^{j \omega_{1} t_{1}}, \ldots, e^{j \omega_{r} t_{N}}\right]=\boldsymbol{d}_{\boldsymbol{r}}
\end{gathered}
$$

$\boldsymbol{q}=\left[q_{1}, q_{2}, \ldots, q_{R}\right]^{T},\left|q_{r}\right|^{2}$ is the power value corresponding to the rth frequency component that is to be calculated.

$\varepsilon=\left[\varepsilon_{1}, \varepsilon_{2}, \ldots, \varepsilon_{N}\right]^{T}$

where $\varepsilon_{1}=\varepsilon_{2}=\cdots=\varepsilon_{N}=\sigma^{2}, \sigma^{2}$ is the noise variance.

The $N \times N$ covariance matrix $\boldsymbol{R}_{N}$ of the received signal is given as

$\boldsymbol{R}_{N}=E\left(\boldsymbol{y y}^{*}\right)=\sum_{r=1}^{R}\left|q_{r}\right|^{2} \boldsymbol{d}_{\boldsymbol{r}} \boldsymbol{d}_{r}^{*}+E\left(\varepsilon \varepsilon^{*}\right)=\boldsymbol{D P D}^{*}$

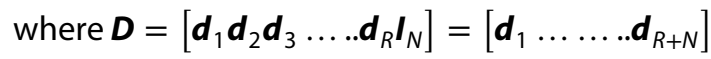

$\boldsymbol{P}=\operatorname{diag}\left(m_{1}, m_{2}, \ldots, m_{R+N}\right)$

where $m_{r}=\left\{\begin{array}{ll}\left|q_{r}\right|^{2}, & r=1,2, \ldots R \\ \sigma^{2}, & r=R+1, R+2, \ldots, R+N\end{array}\right.$;

$E\left(\varepsilon \varepsilon^{*}\right)=\sigma^{2} I_{N}$

The SPICE algorithm is based on minimizing the weighted covariance function $f$.

$f=\left\|\boldsymbol{R}_{N}^{-1 / 2}\left(\hat{\boldsymbol{R}}_{N}-\boldsymbol{R}_{N}\right)\right\|^{2}$

where $\boldsymbol{R}_{N}^{-1 / 2}$ represents the Hermitian square root of $\boldsymbol{R}_{N}^{-1}$, $\|\cdot\|$ denotes the Frobenius norm.

SPICE estimate $[7,8]$ of the $m_{r}{ }^{\prime} s$ is an iterative process of the form:

$m_{r}(i+1)==\frac{m_{r}(i)\left|\boldsymbol{d}_{r}^{*} \boldsymbol{R}_{N}^{-1}(\boldsymbol{i}) \boldsymbol{y}\right|}{w_{r}^{\frac{1}{2}} \rho(i)}$

$w_{r}=\frac{\left\|\boldsymbol{d}_{r}\right\|^{2}}{\|\boldsymbol{y}\|^{2}}$

$\rho(i)=\sum_{s=1}^{R+N} w_{s}^{\frac{1}{2}} m_{s}(i)\left|\boldsymbol{d}_{s}^{*} R_{N}^{-1}(\boldsymbol{i}) \boldsymbol{y}\right|$

where $i$ is the iteration number and $m_{r}(i)$ is the estimate of $m_{r}$ at the ith iteration. The method is initialized with an initial estimate of the $m_{r}$ 's, i.e., $m_{r}(0)=\frac{\left|\boldsymbol{d}_{r}^{*} \boldsymbol{y}\right|^{2}}{\left\|\boldsymbol{d}_{r}\right\|^{4}}$ similar to the periodogram. 
The computational complexity of $\mathcal{O}\left(N^{2} R\right)$ increases with increase in signal points $N$ and frequency domain sampling points $R$. In the next section we introduce a method to reduce the computational complexity of the algorithm.

\section{Implementation of spice algorithm using Gohberg-Semencul Factorization (SPICE GS)}

The computational complexity of the algorithm, to a much extent, depends on the computation of $\boldsymbol{u}=\boldsymbol{R}_{N}^{-1} \boldsymbol{y}$. The direct implementation of SPICE does not take into account the Toeplitz arrangement of Hermitian matrix $\boldsymbol{R}_{N}$. Based on GS factorization [9-13] the inverse of covariance matrix $\mathbf{R}_{\mathrm{N}}$ is denoted by a sequence of the Toeplitz matrices. The matrix product can be enhanced by this factorization. The Levinson-Durbin algorithm (LDA) and the Fast Fourier Transform can be employed to increase the computational speed.

\subsection{Fast computation of the covariance matrix $R_{N}$}

The covariance matrix of observed data is defined as $\boldsymbol{R}_{N}=E\left(\boldsymbol{y y}^{*}\right)=\boldsymbol{D P D}^{*}$.

We rewrite $\boldsymbol{R}_{N}$ as

$$
\begin{aligned}
\mathbf{R}_{N} & =\left[\begin{array}{cccc}
r_{0} & r_{1} & \cdots & r_{N-1} \\
r_{-1} & r_{0} & \cdots & \vdots \\
\vdots & \vdots & \ddots & r_{1} \\
r_{-N+1} & \cdots & r_{-1} & r_{0}
\end{array}\right]=\left[\begin{array}{cccc}
r_{0} & r_{1} & \cdots & r_{N-1} \\
r_{1}^{*} & r_{0} & \cdots & r_{N-2} \\
\vdots & \vdots & \ddots & \vdots \\
r_{N-1}^{*} & r_{N-2}^{*} & \cdots & r_{0}
\end{array}\right] \\
& =\sum_{r=1}^{R+N} m_{r} d_{r} d_{r}^{H}
\end{aligned}
$$

where $r_{-m}=r_{m^{\prime}}^{*} \mathrm{~m}=0,1, \ldots \mathrm{N}-1$.

Each element of the Eq. (15) is specified by

$$
r_{n}=\sum_{r=0}^{K-1} m_{r} e^{-j 2 \pi n r / K}, n=0,1,2, \ldots, N-1 \text { which indi- }
$$

cates that $\left\{r_{m}\right\}_{m=0}^{N-1}$ are the first elements of the

K-point FFT of $\left\{m_{r}\right\}_{r=0}^{K-1}$, where $K=(R+N)$.

Using the first $N$ values of the FFT of $m_{r}, \boldsymbol{R}_{N}$ can be formulated. This requires $\mathcal{O}\left(K \log _{2}(K)\right.$ flops.

\subsection{Fast computation of $R_{N}^{-1} y$}

As soon as the first column of $\boldsymbol{R}_{N}$ is presented, the vector $\boldsymbol{u}$ can be involved in the linear equation $\boldsymbol{R}_{N} \boldsymbol{u}=\boldsymbol{y}$.
The fast computation of $\boldsymbol{R}_{N}^{-1}$ will help to reduce the computational complexity of SPICE algorithm. There are two approaches to compute $\boldsymbol{R}_{N}^{-1}$ taking into account the Hermitian Toeplitz structure of $\boldsymbol{R}_{N}$. The first approach is based on Choleskey decomposition, where the inverse of the correlation matrix of order $N$ is given in terms of all the autoregressive (AR) coefficients up to order $N-1$. The second approach uses Gohberg-Semencul (GS) factorization $[14,15]$ to compute the inverse using only $A R$ coefficients of order $N-1$ which is described below.

\subsubsection{The Gohberg-Semencul Relation}

Let us proceed in the following way to express $\boldsymbol{R}_{N}^{-1}$ only in terms of the coefficients of prediction error filter of order $N-1$ (i.e., $\left.1, a_{N-1}(1), \ldots, a_{N-1}(N-1)\right)$.

First, we built a positive definite Hermitian Toeplitz matrix of size $2 N \times 2 N$.

$\boldsymbol{G}=\left[\begin{array}{cccc:cccc}r_{0} & r_{1} & \cdots & r_{N-1} & r_{N} & r_{N+1} & \cdots & r_{2 N-1} \\ r_{1}^{*} & \ddots & \cdots & r_{N-2} & r_{N-1} & \ddots & \cdots & \vdots \\ \vdots & \vdots & \ddots & \vdots & \vdots & \vdots & \ddots & \vdots \\ r_{N-1}^{*} & \cdots & \cdots & r_{0} & r_{1} & \cdots & \cdots & r_{N} \\ \hdashline r_{N}^{*} & r_{N-1}^{*} & \cdots & r_{1}^{*} & r_{0} & r_{1} & \cdots & r_{N-1} \\ \vdots & \vdots & \cdots & \vdots & \vdots & \vdots & \ddots & \vdots \\ r_{2 N-1}^{*} & \cdots & \cdots & r_{N}^{*} & r_{N-1}^{*} & \cdots & r_{1} & r_{0}\end{array}\right]$

where the values $r_{N}, r_{N+1}, \ldots, r_{2 N-1}$ are recursively computed using Yule-Walker equation

$r_{k}=-\sum_{i=1}^{N-1} a_{N-1}(i) r_{k-1} \quad$ for $k=N, N+1, \ldots, 2 N-1$.

Let us define $\boldsymbol{L}_{1}$ and $\boldsymbol{L}_{2}$ as $N \times N$ lower triangular Toeplitz matrices

$$
\begin{aligned}
& \boldsymbol{L}_{1}=\left[\begin{array}{cccc}
1 & 0 & \cdots & 0 \\
a_{N-1}^{*}(1) & 1 & \cdots & \vdots \\
a_{N-1}^{*}(2) & a_{N-1}^{*}(1) & \cdots & \vdots \\
\vdots & \ddots & \ddots & \vdots \\
a_{N-1}^{*}(N-1) & \cdots & \cdots & 1
\end{array}\right], \\
& \boldsymbol{L}_{2}=\left[\begin{array}{ccccc}
0 & 0 & \cdots & 0 \\
a_{N-1}(N-1) & 0 & \cdots & \vdots \\
a_{N-1}(N-2) & a_{N-1}(N-1) & \cdots & \vdots \\
\vdots & \ddots & \ddots & \vdots \\
a_{N-1}(1) & a_{N-1}(2) & \cdots & 0
\end{array}\right]
\end{aligned}
$$


Let $\boldsymbol{X}$ be $2 N \times 2 N$ matrix, $\boldsymbol{X}=\left[\begin{array}{c:c}\boldsymbol{L}_{1} & O \\ \hdashline \boldsymbol{L}_{2}^{H} & \boldsymbol{A}_{N}\end{array}\right]$

Observe that $(N+1)$ left columns of $\boldsymbol{X}$ have Toeplitz structure, and $\boldsymbol{G} \boldsymbol{X}=\boldsymbol{Y Z}$ where $\boldsymbol{Y}$ is a $(2 N \times 2 N)$ upper triangular matrix with unity elements in its main diagonal and $\boldsymbol{Z}$ is $(2 N \times 2 N)$ diagonal matrix.

$$
\begin{aligned}
\boldsymbol{Z}= & \operatorname{diag}[\epsilon(N-1), \epsilon(N-1), \ldots, \epsilon(N-1), \epsilon(N-2), \\
& \epsilon(N-2), \ldots, \epsilon(1), \epsilon(0)]
\end{aligned}
$$

Since $\boldsymbol{X}^{H}$ and $\boldsymbol{G X}$ are upper triangular and since $\boldsymbol{X}^{H} \boldsymbol{G X}$ is Hermitian, it results in $\boldsymbol{X}^{H} \boldsymbol{G} \boldsymbol{X}=\boldsymbol{Z}$ and $\boldsymbol{G}^{-1}=\boldsymbol{X Z}^{-1} \boldsymbol{X}^{H}$.

Thus

$\boldsymbol{G}^{-1}=\left[\begin{array}{c:c}\boldsymbol{L}_{1} & O \\ \hdashline \boldsymbol{L}_{2}^{H} & \boldsymbol{A}_{N}\end{array}\right]\left[\begin{array}{cccc}\epsilon^{-1}(N-1) & 0 & \cdots & 0 \\ 0 & \epsilon^{-1}(N-1) & \cdots & \vdots \\ \vdots & \vdots & \ddots & \vdots \\ 0 & \cdots & \cdots & \epsilon^{-1}(0)\end{array}\right]\left[\begin{array}{c:c}\boldsymbol{L}_{1}^{H} & \boldsymbol{L}_{2} \\ \hdashline O & \boldsymbol{A}_{N}^{H}\end{array}\right]$
Table 1 Levinson-Durbin algorithm for computing AR coefficients and prediction error

\begin{tabular}{ll}
\hline Step no. & Operation \\
\hline 1. & Initialize the recursion \\
& $a(0)=1, \epsilon(0)=r_{0}$ \\
2. & For $i=0,1, \ldots, N-2$ \\
& $r_{i}=r_{i+1}+\sum_{j=1} a_{i}(j) r_{i-j+1}, \Gamma_{i+1}=-\frac{r_{i}}{\epsilon(i)}$ \\
& For $j=1,2, \ldots, i$ \\
& $a_{i+1}(j)=a_{i}(j)+\Gamma_{i+1} a_{i}^{*}(i-j+1)$ \\
& $a_{i+1}(i+1)=\Gamma_{i+1}$ \\
& $\epsilon(i+1)=\epsilon(i)\left[1-\left|\Gamma_{i+1}\right|^{2}\right]$ \\
\hline
\end{tabular}

$\boldsymbol{G}^{-1}=\left[\begin{array}{c:c}\epsilon^{-1}(N-1) \boldsymbol{L}_{1} \boldsymbol{L}_{1}^{H} & \epsilon^{-1}(N-1) \boldsymbol{L}_{1} \boldsymbol{L}_{2} \\ \hdashline \epsilon^{-1}(N-1) \boldsymbol{L}_{2}^{H} \overline{\boldsymbol{L}}_{1}^{H} & \epsilon^{-1}(N-1) \boldsymbol{L}_{2}^{H} \overline{\boldsymbol{L}}_{2}+\boldsymbol{R}_{N}^{-1}\end{array}\right]$

Since $\boldsymbol{R}_{N}$ and $\boldsymbol{G}$ are Hermitian Toeplitz matrices, they are centro-Hermitian and their inverses are also centro-Hermitian.

Applying this to (18), we obtain

$\epsilon^{-1}(N-1) \boldsymbol{J} \boldsymbol{L}_{1} \boldsymbol{L}_{1}^{H} \boldsymbol{J}=\epsilon^{-1}(N-1)\left(\boldsymbol{L}_{2}^{H} \boldsymbol{L}_{2}\right)^{*}+\left(\boldsymbol{R}_{N}^{-1}\right)^{*}$

where $\boldsymbol{J}$ is a $(N \times N)$ exchange matrix with unity elements in the cross diagonal and zeros elsewhere. Since $\boldsymbol{J}^{2}=\boldsymbol{I}$ and $\boldsymbol{J} \boldsymbol{R}_{N}^{-1} \boldsymbol{J}=\left(\boldsymbol{R}_{N}^{-1}\right)^{*}$

$\epsilon^{-1}(N-1) \boldsymbol{L}_{1} \boldsymbol{L}_{1}^{H}=\epsilon^{-1}(N-1)\left(\boldsymbol{J}\left(\boldsymbol{L}_{2}^{H}\right)^{*} \boldsymbol{J}\right)\left(\boldsymbol{J} \boldsymbol{L}_{2}^{*} \boldsymbol{J}\right)+\boldsymbol{R}_{N}^{-1}$

Using the fact that $\boldsymbol{J}\left(\boldsymbol{L}_{2}^{H}\right)^{*} \boldsymbol{J}=\boldsymbol{L}_{2} \& \boldsymbol{J} \boldsymbol{L}_{2}^{*} \boldsymbol{J}=\boldsymbol{L}_{2}^{H}$

We get $\boldsymbol{R}_{N}^{-1}=\frac{1}{\epsilon(N-1)}\left[\boldsymbol{L}_{1} \boldsymbol{L}_{1}^{H}-\boldsymbol{L}_{2} \boldsymbol{L}_{2}^{H}\right]$

which is the $\mathrm{G}-\mathrm{S}$ relation.

The matrices $\boldsymbol{L}_{1}$ and $\boldsymbol{L}_{2}$ involved in $\boldsymbol{R}_{N}^{-1}$ are Toeplitz matrices, hence $\boldsymbol{R}_{N}^{-1} \boldsymbol{y}$ can be computed using FFT, as a Toeplitz matrix operating on a vector can be viewed as linear convolution operation and linear convolution can be converted into equivalent circular convolution by appropriate modifications to $\boldsymbol{L}_{1}$ and $\boldsymbol{L}_{2}$ matrices and the vector $\boldsymbol{y}$. Thus, $\boldsymbol{R}_{N}^{-1} \boldsymbol{y}$ can be computed with an order of $N \log _{2} N$ flops. The L-D algorithm needs $N^{2}$ flops.
Thus the order of flops needed to compute $\boldsymbol{R}_{N}^{-1} \boldsymbol{y}$ will be $N \log _{2} N+N^{2}$.

The efficient implementation of the SPICE algorithm for MST radar is outlined as follows:

For each iteration of SPICE_GS,

1. Compute the initial power estimate $\left\{m_{r}\right\}$ through $(R+N)$ point FFT of the data vector $\boldsymbol{y}$.

$$
\text { i.e., } m_{r}(i)=|F F T(\boldsymbol{y})|^{2}, r=0,1,2, \ldots, K-1 \text {. }
$$

2. To formulate $\boldsymbol{R}_{N}$, first compute $r_{n}(i)=\sum_{r=0}^{K-} m_{r}(i) e^{j \frac{j \pi n r}{K}}, n=0,1, \ldots, N-1$ which indicates that $\left\{r_{n}\right\}_{n=0}^{N-1}$ are first elements of K-point IFFT of $\left\{m_{r}\right\}_{r=0}^{K-1}$ where $K=R+N$.

Using $r_{n}(i)$ values, $\boldsymbol{R}_{N}$ can be formulated as it is Hermitian Toeplitz matrix.

3. Given $r_{n}, n=0,1, \ldots, N-1, \epsilon(N-1)$, compute AR coefficients of order $(N-1)$ using $L-D$

Algorithm given in Table 1. Using AR coefficients $\boldsymbol{L}_{1}$ and $\boldsymbol{L}_{2}$ matrices can be formulated and

$$
\begin{gathered}
\boldsymbol{u}=\boldsymbol{R}_{N}^{-1} \boldsymbol{y} \quad \text { c a n be computed a s } \\
\boldsymbol{u}=\frac{1}{\epsilon(N-1)}\left[\boldsymbol{L}_{1} \boldsymbol{L}_{1}^{H}-\boldsymbol{L}_{2} \boldsymbol{L}_{2}^{H}\right] \boldsymbol{y} .
\end{gathered}
$$

4. Calculate $w_{r}^{\frac{1}{2}}=\frac{\left\|\boldsymbol{d}_{r}\right\|}{\|\boldsymbol{y}\|}$ and $c_{r}=\left|\boldsymbol{d}_{r}^{*} \mathbf{u}\right|, \rho=\sum_{l=1}^{R+N} w_{l}^{\frac{1}{2}} m_{l} c_{l}$.

5. Update the power values $m_{r} \leftarrow m_{r} c_{r} / w_{r}^{\frac{1}{2}} \rho$ till the convergence criterion is met. 


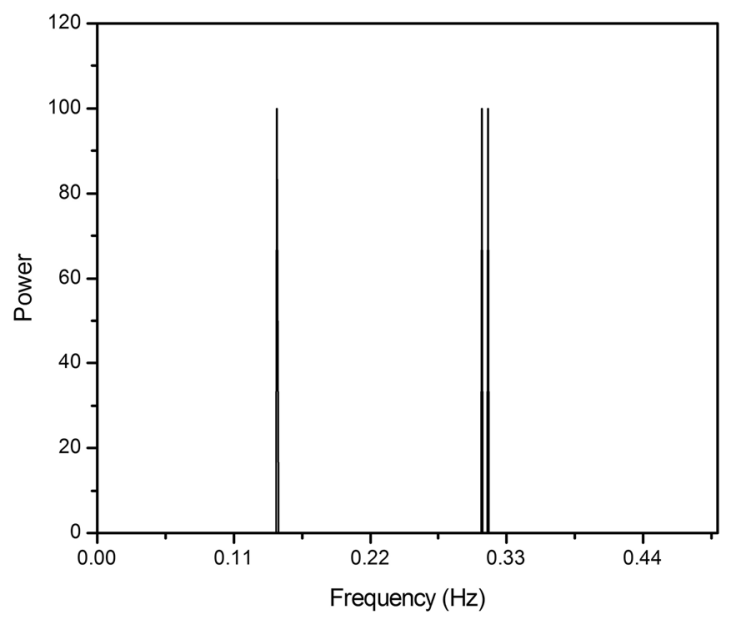

(a)

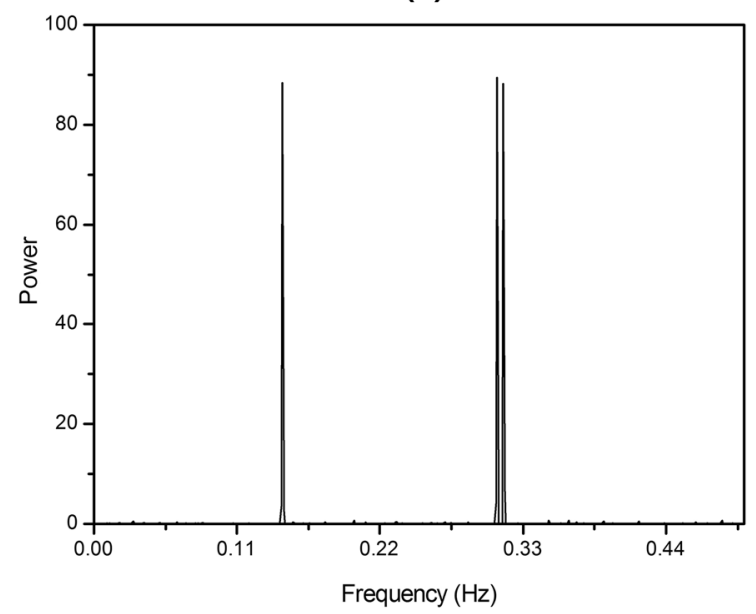

(c)

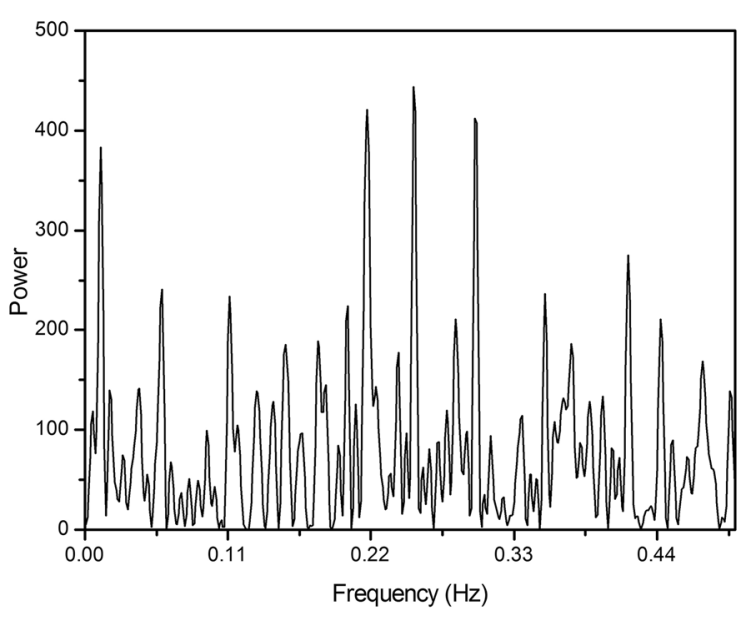

(b)

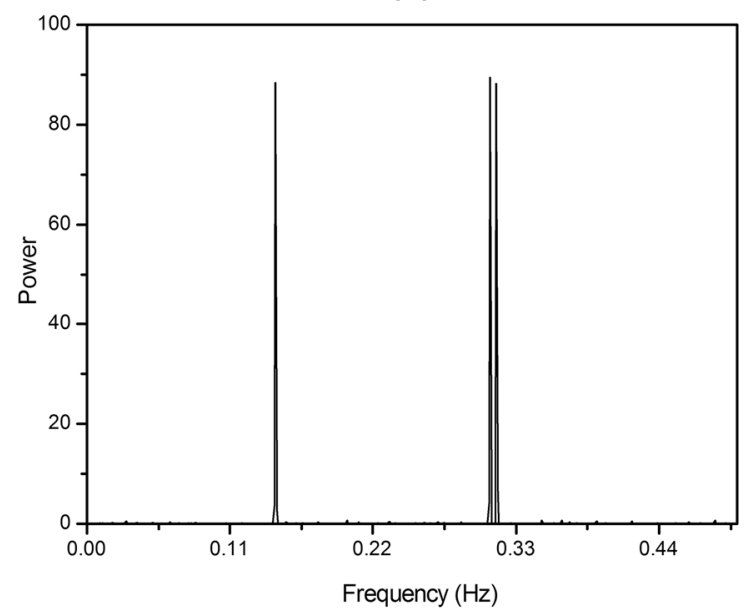

(d)

Fig. 1 a Spectrum of the original signal; $\mathbf{b}$ power Spectrum using periodogram; c SPICE; d SPICE_GS

Table 2 The main operation process of SPICE and SPICE_GS

\begin{tabular}{lll}
\hline Method & Step & $\begin{array}{l}\text { Compu- } \\
\text { tational } \\
\text { complexity }\end{array}$ \\
\hline SPICE & Covariance matrix calculation & $N^{2} R$ \\
& Inverse covariance matrix & $N^{2} R$ \\
SPICE_GS & Covariance matrix calculation & $R \log _{2} R$ \\
& Inverse covariance matrix & $N^{2}+N \log _{2} N$ \\
\hline
\end{tabular}

\section{Results}

\subsection{Simulation results}

The examples are presented for simulated data to examine the computational complexity between direct and the
Table 3 Computational complexity of the proposed and existing methods for various number of data sample points

\begin{tabular}{lrl}
\hline $\begin{array}{l}\text { No. of } \\
\text { data } \\
\text { points }\end{array}$ & \multicolumn{2}{l}{ Time in seconds } \\
\cline { 2 - 3 } & SPICE & SPICE_GS \\
\hline 64 & 21.33 & 0.75 \\
128 & 29.62 & 0.86 \\
256 & 51.23 & 0.92 \\
512 & 72.97 & 1.61 \\
1024 & 138.89 & 1.73 \\
4096 & 265.61 & 1.91 \\
\hline
\end{tabular}

proposed fast implementation of SPICE. The simulation parameters considered are as follows:

With $\mathrm{N}=200$ and $\mathrm{C}=3$, the data samples are generated, which contains the three exponentials at $0.3100 \mathrm{~Hz}$, $0.3150 \mathrm{~Hz}$ and $0.1450 \mathrm{~Hz}$, having amplitudes $q_{1}=10 e^{j \varphi_{1}}$, $q_{2}=10 e^{j \varphi_{2}}$, and $q_{3}=10 e^{j \varphi_{3}}$ with a interval of $1 \mathrm{~s}$. The phase values $\left\{\varphi_{r}\right\}_{r=1}^{3}$ are uniformly distributed in the range [0, 
Fig. 2 Height profiles of SNR estimated using ADP and SPICE_GS for data obtained on Feb 9, 2015. a The east beam and $\mathbf{b}$ the south beam

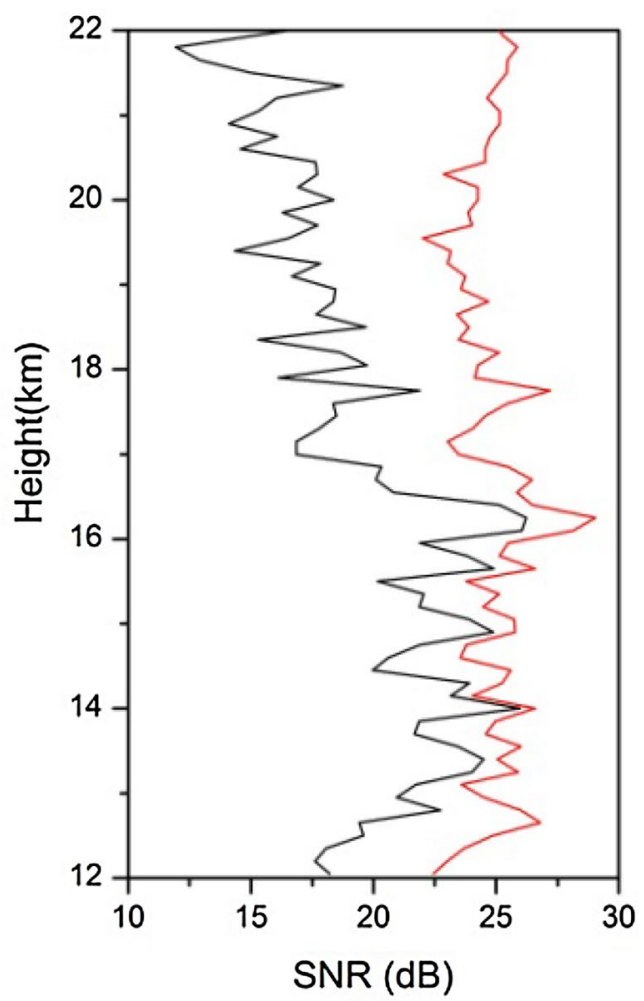

(a)

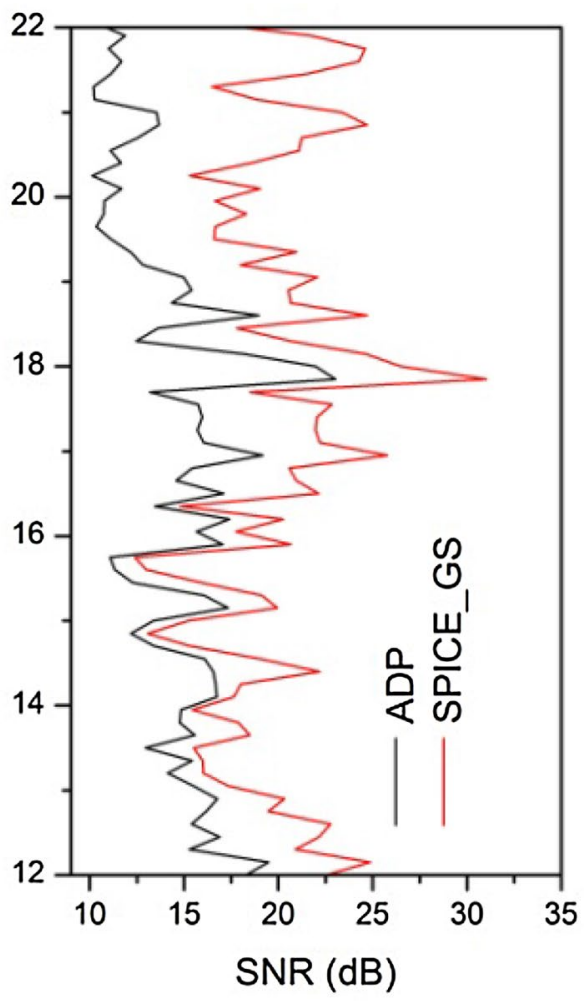

(b)
$2 \pi$ ]. The AWGN with zero mean and variance $\sigma$ is added in the $\varepsilon$ term.

The spectrum of the original signal before adding noise, computed using periodogram, implemented by SPICE and also implemented by fast SPICE_GS for SNR $=-15 \mathrm{~dB}$ are shown in Fig. 1a-d respectively. It is apparent from the simulations, though the signal is entirely concealed in the noise, the SPICE methods, both direct implementation as well as Fast implementation, are able to estimate the parameters well. The periodogram is attained by padding 200 time series data points with 312 zeros and calculating the 512-point FFT. The number of points for processing for both techniques is 512 .

The output SNR is estimated from the spectrum of the signal utilizing the noise level estimation method [16]. This is the widely used technique for noise threshold estimation and removal in atmospheric radar.

The simulations are carried on Intel Core i7-6700 CPU 3.4 GHz with $8 \mathrm{~GB} C P U$ memory. The comparison of the main operation process of SPICE and SPICE_GS are tabulated in Table 2. The computational complexity attained by the proposed method over the existing is illustrated in Table 3, for various data points.

\subsection{Results for MST radar data}

The radar data is gathered from the "Indian MST radar", which is operated at the NARL, Gadanki, Andhra Pradesh. This time-series data is subjected to the ADP, which normally uses the method of periodogram for the calculation of power spectrum. The output SNR estimated using ADP and SPICE_GS for east and south beams are depicted in Fig. 2.

Figure 3 shows the power spectrum at the range bins numbered 63 estimated using ADP and SPICE_GS for the east beam of Feb 9,2015 data. SPICE-GS identifies the particular frequency than the ADP which demonstrates the accuracy of the algorithm.

The zonal wind $v_{x}$, meridional wind $v_{y}$, and wind speed W components obtained using SPICE_GS, ADP and GPS radiosonde for the data on Feb 09, 2015, are depicted in the Fig. 4. From Fig. 4, it can be seen that the proposed SPICE_GS is following the GPS, especially in the range 14-17 km. However, the wind profile curves obtained using the ADP deviate from those obtained using GPS, in a considerable measure, after a height of $11 \mathrm{~km}$. The direct implementation SPICE and the fast implementation SPICE_GS yields the same results and so we have shown the fast implementation results.

The comparison of the computational time of direct and fast implementation of SPICE for radar data processing is 
Fig. 3 Spectrum at bin 63 of the east beam for data obtained on Feb 9, 2015. a ADP and b SPICE_GS

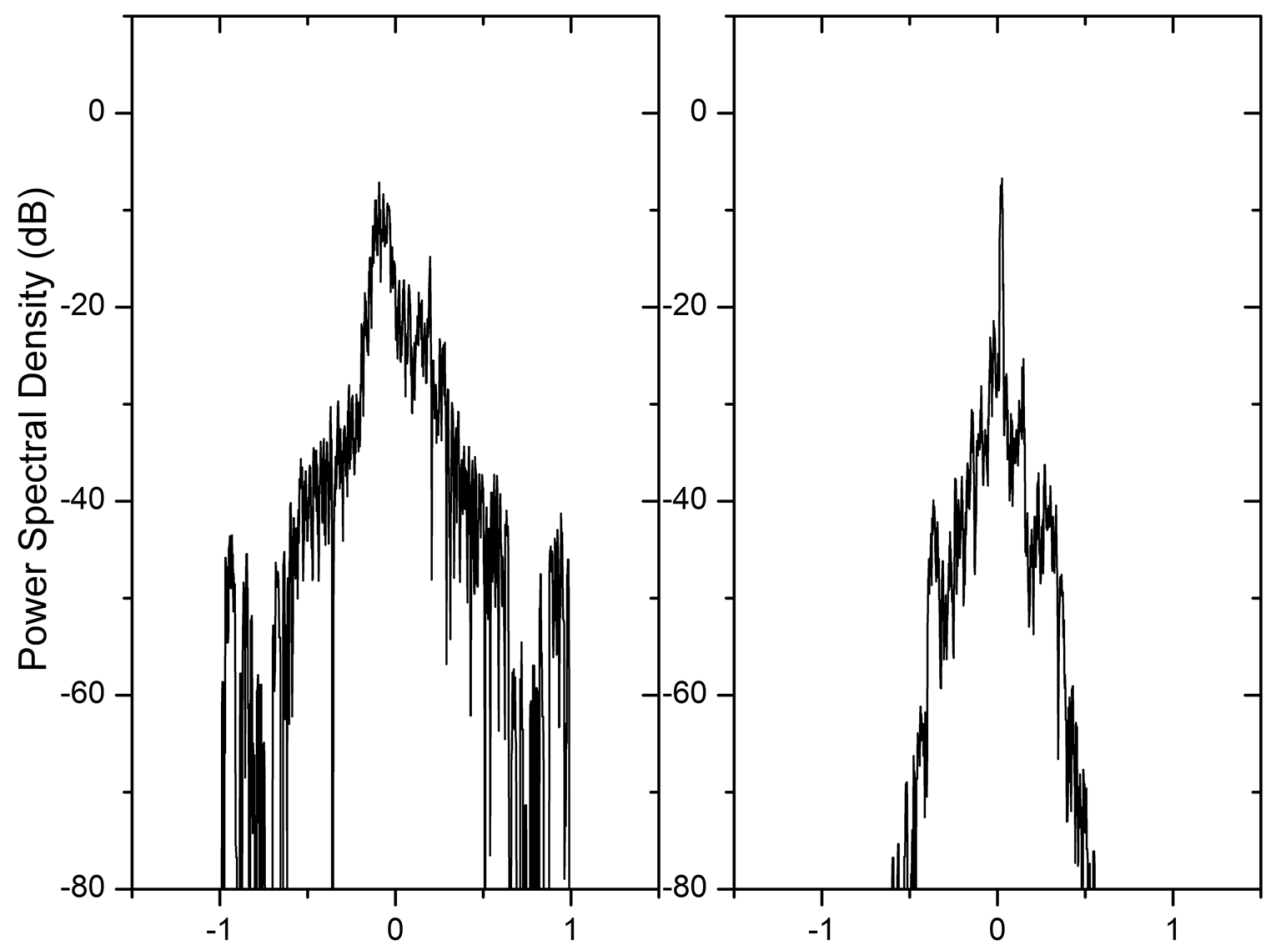

(a)

Normalised Frequency $(\mathrm{Hz})$

Fig. 4 Zonal, meridional and wind speed comparing GPS radiosonde, SPICE_GS and ADP
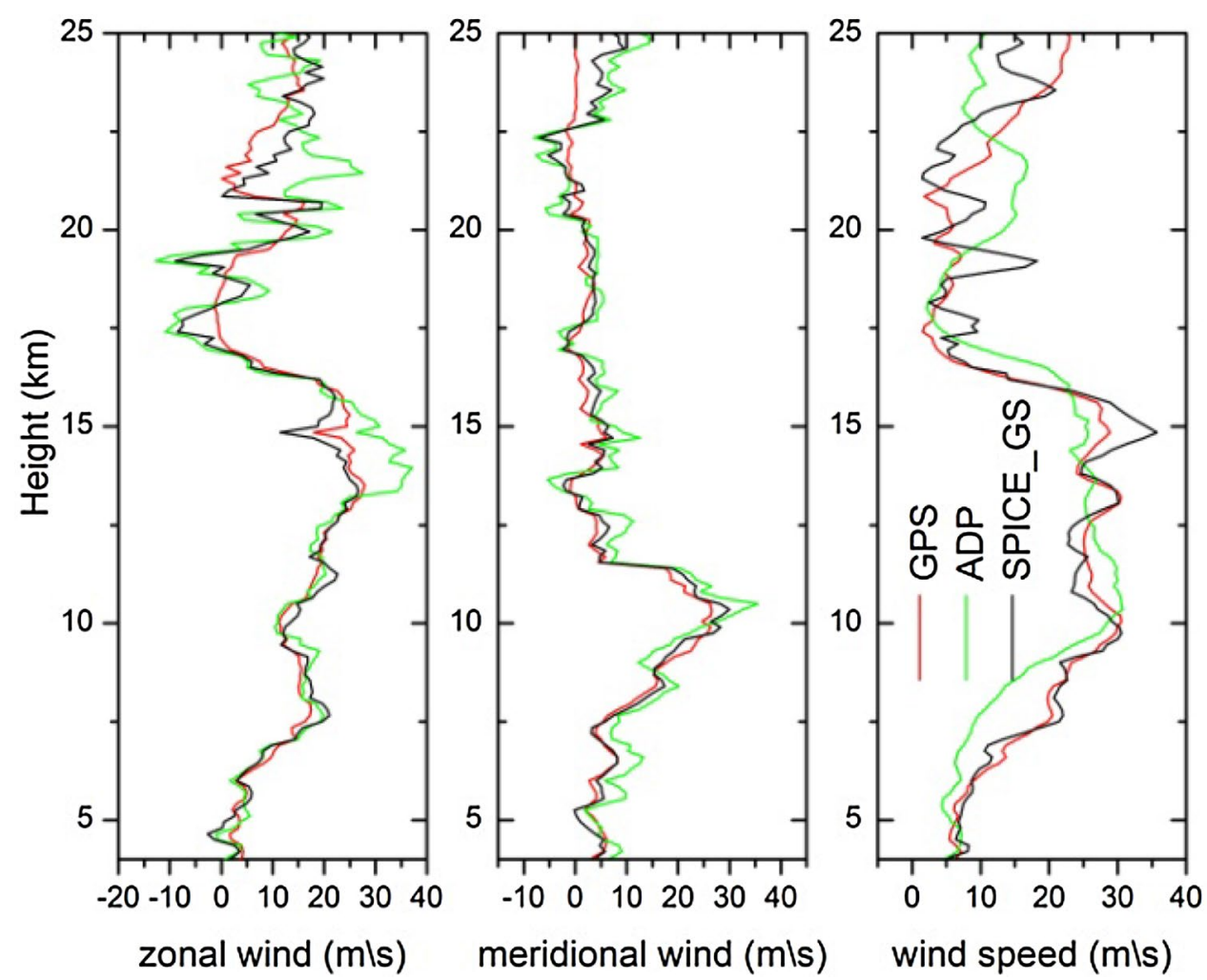


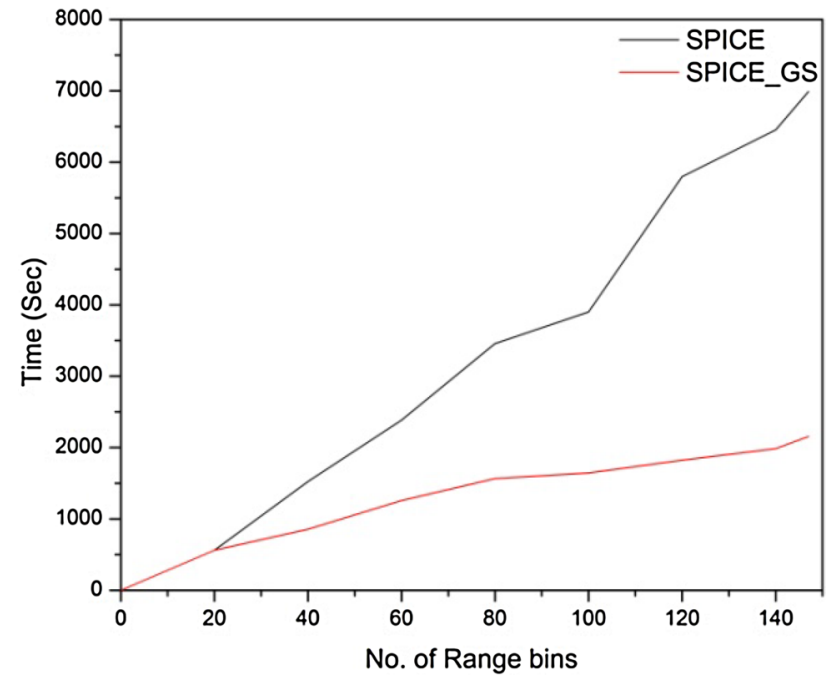

Fig. 5 Computational time comparison plot for processing MST radar data by SPICE and SPICE_GS

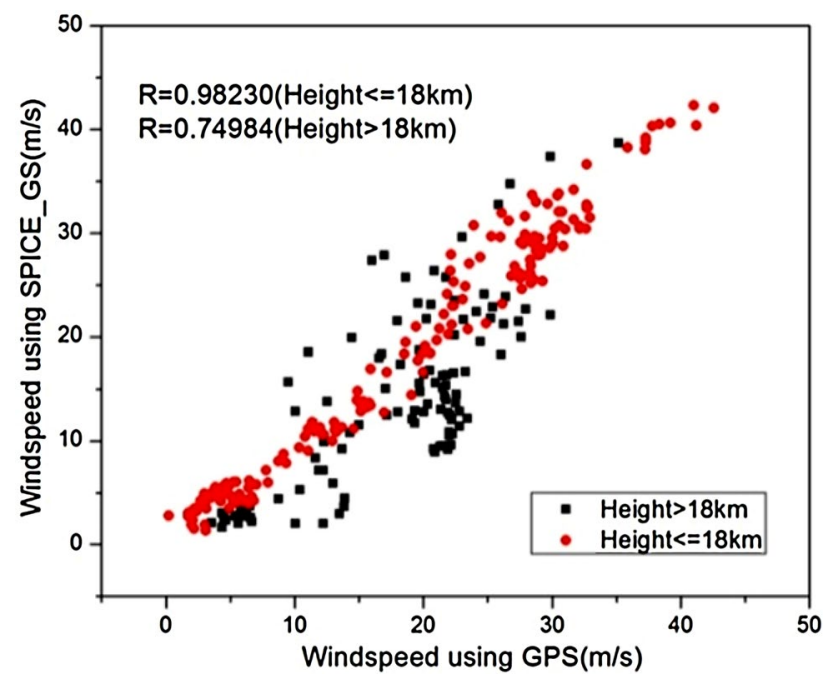

Fig. 6 Correlation between GPS wind speed and SPICE_GS wind speed

shown in Fig. 5 portraying a substantial time reduction in fast implementation than that of direct implementation, making the former striking.

Suitability of the method for processing the MST radar data is tested by correlation studies. The correlation between the GPS radiosonde data and SPICE_GS wind speed is displayed in Fig. 6 for the radar data retrieved during Feb 09-12, 2015. The correlation factor for height less than or equal to $18 \mathrm{~km}$ is 0.98230 and that for height greater than $18 \mathrm{~km}$ is 0.74984 .

\section{Conclusion}

The fast implementation of semiparametric method for spectrum estimation, i.e., SPICE_GS, is applied to MST radar data retrieved from the NARL. Since the Gohberg-Semencul factorization of the covariance matrices is applied, we can leverage the Toeplitz/blockToeplitz structure to compute the spectral estimate. The processed results have revealed noteworthy computational reductions. A considerable increase in computational efficiency has been obtained by SPICE_GS, devoid of the loss in performance.

Acknowledgements The authors thank National Atmospheric Research Laboratory (NARL), Gadanki for giving the radar data and CoE, ECE Department, SVUCE for technical assistance.

\section{Compliance with ethical standards}

Conflict of interest The author states that, there is no conflict of interest.

\section{References}

1. Stoica P, Moses RL (2005) Spectral analysis of signals. PrenticeHall, Upper Saddle River, NJ

2. Stoica P, Babu P, Li J (2011) New method of sparse parameter estimation in separable models and its use for spectral analysis of irregularly sampled data. IEEE Trans Signal Process 59(1):35-47

3. Thatiparthi S, Gudheti R, Sourirajan V (2009) MST radar signal processing using wavelet-based denoising. IEEE Geosci Remote Sens Lett 6(4):752-756

4. Reddy T, Reddy GR (2010) MST radar signal processing using cepstral thresholding. IEEE Trans Geosci Remote Sens 48(6):2704-2710

5. Reddy TS, Reddy GR (2010) Spectral analysis of atmospheric radar signal using filter banks polyphase approach. Digit Signal Process 20(4):1061-1071

6. Rao DUM, Reddy TS, Reddy GR (2014) Atmospheric radar signal processing using principal component analysis. Digit Signal Process 32:79-84

7. Eappen NI, Sreenivasulu Reddy T, Ramachandra Reddy G (2016) Semiparametric algorithm for processing MST radar data. IEEE Trans Geosci Remote Sens 54(5):2713-2721

8. Raju C, Sreenivasulu Reddy T (2018) Sparse iterative covariance based estiamtion for atmospheric radar data. Int J Eng Technol (UAE) 7(1):232-236

9. Gohberg IC, Semencul AA (1972) On the inversion of finite Toeplitz matrices and their continuous analogs. Mat Issled 2:201233 (in Russian)

10. Huckle T (1993) Computation of Gohberg-Semencul formula for a Toeplitz matrix. Technical report, University Wurzburg and Stanford University

11. Xue M, Xu L, Li J (2011) IAA spectral estimation: fast implementation using the Gohberg-Semencul factorization. IEEE Trans Signal Process 59(5):3251-3261

12. Hang Q, Abedia H, Xue M, Rowe W, Li J (2012) Fast Implementation of sparse iterative covariance-based estimation for source localization. J Acoust Soc Am 131(2):1249-1259 
13. Jiang Xiaoyu, Hong Kicheon (2017) Gohberg-Semencul type formula and application for the inverse of a conjugate-Toeplitz matrix involving imaginary circulant matrices. J Nonlinear Sci Appl 10:2848-2859

14. Zheng Y, Shon S, Fu Z (2018) A Gohberg-Semencul type formula for the inverse of conjugate-Toeplitz matrix and applications. Acta Math Appl Sin Engl Ser 34:293-303

15. Jiang Z-L, Chen J-X (2017) The explicit inverse of nonsingular conjugate-Toeplitz and conjugate-Hankel matrices. J Appl Math Comput 53:1-16
16. Hildebrand $\mathrm{PH}$, Sekhon $\mathrm{R}$ (1974) Objective determination of the noise level in Doppler spectra. J Appl Meteorol 13(7):808-811

Publisher's Note Springer Nature remains neutral with regard to jurisdictional claims in published maps and institutional affiliations. 